\title{
Recovery of photoreceptor outer segments after anti-VEGF therapy for age-related macular degeneration.
}

\section{AUTHOR(S):}

Oishi, Akio; Shimozono, Masataka; Mandai, Michiko; Hata, Masayuki; Nishida, Akihiro; Kurimoto, Yasuo

\section{CITATION:}

Oishi, Akio ...[et al]. Recovery of photoreceptor outer segments after anti-VEGF therapy for age-related macular degeneration.. Graefe's archive for clinical and experimental ophthalmology 2013, 251(2): 435-440

\section{ISSUE DATE:}

2013-02

URL:

http://hdl.handle.net/2433/189745

\section{RIGHT:}

The final publication is available at Springer via http://dx.doi.org/10.1007/s00417-0122034-4; この論文は出版社版でありません。引用の際には出版社版をご確認ご利用くだ さい。; This is not the published version. Please cite only the published version. 
Recovery of OCT findings after the treatment of AMD

Recovery of photoreceptor outer segments after anti-VEGF therapy for age-related macular degeneration

Akio Oishi, ${ }^{1,2}$ Masataka Shimozono, ${ }^{1}$ Michiko Mandai, ${ }^{1,3}$ Masayuki Hata, ${ }^{1}$ Akihiro Nishida, ${ }^{1}$ Yasuo Kurimoto, ${ }^{1}$

${ }^{1}$ Department of Ophthalmology, Kobe City Medical Center General Hospital.

${ }^{2}$ Department of Ophthalmology and Visual Sciences, Kyoto University Graduate School of Medicine.

${ }^{3}$ Laboratory for Retinal Regeneration, Center for Developmental Biology, RIKEN.

Correspondence to Akio Oishi, MD, PhD, Department of Ophthalmology, Kobe City Medical Center General Hospital, 4-6 Minatojima-minamimachi, Chuo-ku, Kobe 650-0046, Japan.

Telephone: (81)-78-302-4321; fax: (81)-78-302-2487

E-mail: aquio@kuhp.kyoto-u.ac.jp

The authors have no proprietary interest in any aspect of this study. 
Recovery of OCT findings after the treatment of AMD

Purpose: To evaluate whether the status of the external limiting membrane (ELM) or inner segment/outer segment junction (IS/OS) improves after intravitreal injection of ranibizumab for age-related macular degeneration (AMD). We also evaluated whether the pre-operative values of these parameters are associated with the visual prognosis.

Methods: Hospital-based, cross-sectional study. 76 eyes of 76 treatment naïve AMD patients who received three monthly intravitreal injections of ranibizumab followed for more than six months with additional as-needed injections were investigated. Spectral domain OCT was used to evaluate the length of ELM, IS/OS, and foveal thickness pre- and post-operatively. Changes of ELM and IS/OS length were evaluated postoperatively. Correlation coefficients between pre-operative parameters and post-operative visual acuity were also analyzed.

Results: Significant changes were noted in mean logMAR (0.66 to 0.53), foveal thickness (231.1 to $151.1 \mu \mathrm{m})$, and IS/OS length (514.9 to $832.3 \mu \mathrm{m})$ after the treatment. ELM length did not improve significantly (1312.4 to $1376.7 \mu \mathrm{m})$. Although pre-operative ELM length, IS/OS length, and foveal thickness showed correlation with post-operative $\log \mathrm{MAR}(R=-0.51,-0.39$, and 0.46 , respectively), 
Recovery of OCT findings after the treatment of AMD

the most powerful predictive factor for visual prognosis was pre-operative

$\log M A R(R=0.77, P<0.001)$

Conclusions: IS/OS status improves in response to anti-VEGF therapy but

ELM seems to have less plasticity. Restoration of IS/OS occurs where ELM is

retained. The status of IS/OS and ELM can be used as prognostic factors but the predictive power is inferior to that of baseline visual acuity. 
Recovery of OCT findings after the treatment of AMD

\section{Introduction}

Optical coherence tomography (OCT) is widely used to assess microstructural changes in the retina, especially that of macular pathologies. Since the era of time-domain OCT, which had limited axial resolution of up to $10 \mu \mathrm{m}$, the device has been a standard tool in clinical practice[1] and in the management of age-related macular degeneration (AMD).[2-5] Moreover, currently available spectral-domain OCT machines achieve up to 5- $\mu \mathrm{m}$ resolution and allow layer-by-layer evaluation of the retina.[6, 7]

In AMD, photoreceptor integrity, as indicated by the retinal layer pattern, is important for vision. Several groups have shown that continuity of the junction between inner and outer segments of the photoreceptors (IS/OS) is associated with good visual acuity (VA) in AMD patients who were treated with PDT[8] or with anti-VEGF agents. [9] Another group used microperimetry and confirmed the association of IS/OS status and retinal sensitivity.[10] Our group has reported that intact ELM as well as IS/OS is important for good vision. [11] Evaluation of these retinal changes provides detailed information and more profound understanding of retinal pathologies.[12]' [13] The treatment of AMD drastically changed after two pivotal studies showed 
Recovery of OCT findings after the treatment of AMD

marked effects of anti-vascular endothelial growth factor (VEGF) therapy.[14-16]

The treatment enabled not only maintenance of vision but also improvement in patients with any subtype of AMD over a 24-month period.[14-16] Although the relatively high cost of the drug and the need for ongoing intravitreal injections are demanding,[17] no other treatment has achieved these levels of visual acuity gain[18] and thus the treatment is now a first-line choice for AMD.

Given the improvement in vision, it is clear that anti-VEGF therapy is beneficial for the retina. However, how the therapy affects retinal microstructures such as IS/OS or ELM has not been evaluated. Evaluation of these microstructures would provide more detailed information about the retinal changes during the treatment course and could help identify the potential for plasticity of these structures, i.e., how much photoreceptor damage could be recovered with the current standard treatment regimen. In addition, if the morphologic changes during the treatment process are consistent, we may be able to predict visual prognosis from pre-operative findings because post-operative retinal morphology is associated with post-operative vision. $[8,9,11]$ In the present study, we investigated quantitatively the changes of IS/OS and ELM after treatment. We also examined pre-operative findings and visual 
Recovery of OCT findings after the treatment of AMD

prognosis to test whether these morphological features can be used as predictive factors.

\section{Methods}

All procedures conformed to the tenets of the Declaration of Helsinki and the study design was approved by the institutional review board of Kobe City Medical Center General Hospital. The review board waived the need for written informed consent because the study design consisted of retrospective chart review.

We retrospectively reviewed the clinical records of patients who underwent three monthly injections of ranibizumab for AMD from February 2009 to February 2011 and were followed for more than six months at Kobe City Medical Center General Hospital. Diagnosis of AMD was made with funduscopy, slitlamp examination, OCT, and fluorescein / indocyaningreen angiography. Patients who were under 50 years old or who had refractive error less than -6 diopters or axial length more than $26.5 \mathrm{~mm}$ were not considered as having AMD. Only patients who were examined with SD-OCT before and after a follow-up period of more than six months were included in the study. Exclusion criteria included 
Recovery of OCT findings after the treatment of AMD

other retinal or optic nerve diseases such as retinal vein occlusion, diabetic retinopathy, or optic neuropathy. Those who were previously treated with photodynamic therapy or laser photocoagulation and who dropped out from the treatment regimen were also excluded. SD-OCT images were obtained with Spectralis: Spectralis Family Acquisition Module (version 4.0.2.0, Heidelberg Engineering, Dossenheim, Germany) with Heidelberg Eye Explorer (version 1.6.1.0). Horizontal scans of the macula were recorded for each eye. The measurements were performed under pupillary dilation. The eye tracking ART (automatic real time) software of the device was used to assure that the scans made were in the correct position and averagings were properly done; the position of the fovea was manually detected during examination. We routinely averaged 60 scans per image and when the examination failed to average more than 40 images or to scan the foveola due to poor fixation or media opacity, the patients were excluded from the study.

We measured the length of ELM and IS/OS within regions of $1 \mathrm{~mm}$ nasal and temporal from the fovea (Figure 1). Measurement of ELM and IS/OS length was manually done using software built in the OCT. To investigate the diagnostic value of qualitative evaluation, whether each line is continuous or 
Recovery of OCT findings after the treatment of AMD

disrupted at fovea were also investigated. Foveal thickness was defined as the distance between the vitreoretinal border and the edge of the retinal pigment epithelium. When retinal detachment or sub-retinal fibrosis was seen, the inner border of the subretinal fluid or fibrosis was used as the outer boundary. To evaluate the reproducibility and inter-examiner variability for the measurements, two independent examiners (A.O. and M.S.), who were masked as to the VA of the study participants, measured the above parameters and inter-observer variation was calculated. The average of two measures was used for the statistical analysis. In addition, images from 20 randomly selected patients were re-measured by the same examiner (M.S.) two months after the first measurement and intra-observer variability was calculated. Clinical characteristics, including age, sex, and angiographic classification: occult with no classic, minimally classic, and predominantly classic were also reviewed and analyzed. To detect maximal changes after the treatment, the image with least exudative changes was selected for each patient and evaluated as the post-operative image. Selection of the images with least exudative changes was done by two of the authors (A.O. and M.S.) and always coincided. Statistical analysis was performed using IBM SPSS Statistics Desktop (version 
Recovery of OCT findings after the treatment of AMD

19.0.0; IBM Japan, Tokyo, Japan). Descriptive analyses are reported as means \pm standard deviation unless otherwise specified. Visual acuities were measured with a Landolt $\mathrm{C}$ chart and then converted to logarithm of the minimum angle of resolution (logMAR) equivalents. Bivariate relationships were examined using Spearman's rank correlation coefficient. Intra and inter examiner variability was tested using intraclass correlation coefficients $\operatorname{ICC}(1,1)$ and $\operatorname{ICC}(2,1)$, respectively. Group means for each measurement were compared using one-way analysis of variance (ANOVA) with Bonferroni correction. $P$ values of less than 0.05 were considered to be statistically significant.

\section{Results}

We reviewed 144 eyes of 144 patients who were planned to undergo three monthly induction and as-needed additional treatment. After the exclusion of 11 patients with poor quality image, 45 patients with previous treatment, and 12 patients who dropped out from the treatment regimen, a total of 76 eyes of 76 patients with AMD met the inclusion criteria. The mean age of the enrolled patients was $77.3 \pm 9.6$ years; there were 45 men and 31 women. Pre-operative logMAR was $0.67 \pm 0.41$. Thirty-four eyes were classified as occult with no classic, 17 as minimally classic, and 25 as predominantly classic. 
Recovery of OCT findings after the treatment of AMD

Foveal thickness was $231.1 \pm 136.2 \mu \mathrm{m}$. The ELM length was $1312.4 \pm 660.3$ $\mu \mathrm{m}$ and the IS/OS length was $514.9 \pm 574.2 \mu \mathrm{m}$. The inter-examiner measurements were highly correlated; intraclass correlation coefficients between the measurements by two of the authors were 0.84 for ELM and 0.89 for IS/OS. Intra-examiner correlation coefficients were 0.85 for ELM and 0.93 for IS/OS.

IS/OS was disrupted at fovea in 56 cases and ELM in 30 cases. Disruption of each line at fovea was associated with poor logMAR $(0.76 \pm 0.40$ in IS/OS disrupted and $0.39 \pm 0.31$ in IS/OS continuous cases, $P<0.001 ; 0.92 \pm 0.41$ in ELM disrupted and $0.49 \pm 0.31$ in ELM continuous cases, $P<0.001)$ ELM was almost always (74 of 76 cases) longer than IS/OS; when IS/OS was preserved, they were almost always accompanied by ELM. Those with occult with no classic lesion had longer ELM (1580.6 $\pm 516.9 \mu \mathrm{m})$ and IS/OS (677.3 \pm 646.2 $\mu \mathrm{m})$ compared to those with minimally classic lesion (ELM:1115.3 $\pm 657.8 \mu \mathrm{m}$, $\mathrm{P}=0.04$; IS/OS:258.1 $\pm 422.2 \mu \mathrm{m}, \mathrm{P}=0.04$ ) or predominantly classic lesion (ELM:1081.7 $\pm 722.4 \mu \mathrm{m}, \mathrm{P}=0.01 ; \mathrm{IS} / \mathrm{OS}: 468.7 \pm 501.0 \mu \mathrm{m}, \mathrm{P}=0.48)$ The participants underwent $4.2 \pm 1.2$ intravitreal injections during the observation period of $7.7 \pm 1.9$ months. LogMAR improved to $0.53 \pm 0.41$. Foveal 
Recovery of OCT findings after the treatment of AMD

thickness decreased to $151.1 \pm 48.5 \mu \mathrm{m}, \mathrm{P}<0.001$, probably reflecting the resolution of retinal edema.[19](Figure 2) IS/OS length increased to $832.3 \pm$ $743.3 \mu \mathrm{m}, \mathrm{P}<0.001$. In other words, the IS/OS recovered approximately $60 \%$ of its continuity.(Figure 3) On the other hand, post-operative ELM length was $1376.7 \pm 654.6 \mu \mathrm{m}$ and was not significantly different from pre-operative measurements. There was no significant difference in ELM and IS/OS improvements (post-operative length minus pre-operative length) among occult and classic lesions. Foveal disruption of IS/OS $(n=46)$ and ELM $(n=26)$ was again associated with visual acuity $(0.74 \pm 0.37$ in IS/OS disrupted and $0.21 \pm$ 0.21 in IS/OS continuous cases, $\mathrm{P}<0.001 ; 0.94 \pm 0.34$ in ELM disrupted and 0.31 \pm 0.25 in ELM continuous cases, $P<0.001$ ). Pre-operative or post-operative foveal disruption of ELM or IS/OS made no difference in ELM or IS/OS improvement.

Since the mean ELM length did not show significant changes, we further assessed the status of the ELM in individual cases. We reviewed the images of 19 eyes that showed more than $300-\mu \mathrm{m}$ improvement of the ELM (based on the standard deviation SD of the difference between the two observers: $289.9 \mu \mathrm{m}$ ). These cases, in the pre-operative state, typically showed abnormalities in the 
Recovery of OCT findings after the treatment of AMD

outer retina that could complicate the interpretation (Figure 4). In fact, 12 cases had subretinal fibrin like material, 4 cases had CME, 2 cases had subretinal fibrovascular lesion, and 2 cases had accumulation of exudates (some cases had combinations of these). We cannot tell whether the ELM had defects or if the ELM was only invisible due to these masking factors in these cases.

We also investigated 38 eyes, which showed more than 300- $\mu \mathrm{m}$ IS/OS restoration. Fibirin, $\mathrm{CME}$, subretinal fibrovascular lesion, and/or accumulation of exudates were also noted in these eyes (16, 10, 2, and 6 eyes, respectively). The influences of these masking factors were again suspected in these cases. In addition, IS/OS restoration was observed only in area where ELM existed post-operatively but pre-operative ELM preservation was not a pre-requisite for IS/OS recovery.

We then assessed the prognostic value of these parameters. Pre-operative foveal thickness, ELM length, and IS/OS length were associated with post-operative $\log M A R(R=0.46, P<0.001 ; R=-0.51, P<0.001 ; R=-0.39, P=$ 0.001; respectively). However, pre-operative logMAR showed a stronger correlation $(R=0.77, P<0.001)$. When we performed a multiple linear-regression analysis, factors other than pre-operative logMAR did not show 
Recovery of OCT findings after the treatment of AMD

significant effects on post-operative logMAR. The adjusted $\mathrm{R}^{2}$ for the model was 0.59 ; the standardized regression coefficient of pre-operative logMAR was $0.77, \mathrm{P}<0.001$.

\section{Discussion}

We evaluated the changes of OCT-depicted retinal structures, especially ELM and IS/OS, after anti-VEGF therapy. The results indicated that the IS/OS improves in response to the treatment; however, ELM has less plasticity. The study also showed the prognostic potential of these factors, although the predictive power seemed inferior to that of pre-operative visual acuity. Anti-VEGF therapy is now a first-line treatment for AMD.[20] With the treatment regimen, retinal thickness improved and some of the IS/OS disturbance recovered. The plastic capacity of the IS/OS is plausible considering that outer segments reproduce daily and it takes less than 10 days for complete renewal.[21] In addition, the recovery of IS/OS always occurred where ELM existed. The finding confirms the notion that healthy photoreceptor is needed to produce outer segments and presence of ELM can be an indicator of photoreceptor integrity. It would be interesting to see the notion is applicable in other diseases. 
Recovery of OCT findings after the treatment of AMD

On the other hand, ELM seemed to have limited plasticity; ELM length did not change significantly and false-positive effects were frequently suspected in the individual cases where ELM improved. The result suggested that ELM disruption indicates more severe damage to photoreceptor cell bodies and it should be more difficult, if possible as we reported in closed $\mathrm{MH}$ [22], to overcome. Given that we do not have a way to directly regenerate the damaged retina, treatment should be as early as possible to avoid irreversible changes in the photoreceptors.

Predicting the visual prognosis from pre-operative OCT findings is of clinical interest in the management of AMD.[23-26] In the present study, foveal disruption of each line was associated with visual acuity in pre-operative or post-operative state in consistent with the previous reports. [8-11] In addition, we showed that foveal thickness, length of ELM, and length of IS/OS have prognostic value for vision. However, pre-operative visual acuity showed stronger correlation with visual prognosis, consistent with the previous notion that OCT or angiography alone does not offer sufficient prediction of function.[27] The result from the present multiple regression analysis suggested that pre-operative OCT findings are confounding factors of 
Recovery of OCT findings after the treatment of AMD

pre-operative logMAR. Thus, although we can roughly predict visual prognosis from OCT findings, checking pre-operative visual acuity seems to be the most efficient way at present. Some modifications including refinement of image resolution[28] or combination of parameters would be needed for practical use. There are several limitations to the present study including the retrospective study design, a relatively small population from a single institution, and the use of the Landolt $\mathrm{C}$ chart for VA measurement rather than the ETDRS chart used in major clinical trials. Another limitation is the very nature of OCT examinations. The device depicts only a difference in light reflectance. Thus, disturbance of the ELM or IS/OS detected in OCT images does not necessarily indicate the disturbance of photoreceptors in a histopathologic context. We should keep in mind that these features are just parameters and not necessarily anatomic changes. Clinical relevance and histologic interpretation of the findings should be considered differently.

Additionally, there were difficulties in manual measurement of ELM or IS/OS length. When compared to more orderly images as seen in epiretinal membrane $(E R M)$ or macular hole $(\mathrm{MH})$, AMD-affected retinas often were accompanied by fibrin, hemorrhage, or hard exudates, which make the 
Recovery of OCT findings after the treatment of AMD

interpretation of retinal structures difficult. The improvement of these lines can be a result of better visualization rather than histologic recovery. In addition, the images are often not in alignment due to subretinal fluid or hemorrhage. Although we confirmed the inter- and intra-observer reproducibility of the results, measurements were less consistent when compared to our previous experience dealing with ERM[29] or $\mathrm{MH} .[22]$

Finally, we have shown that the IS/OS improves in response to treatment but ELM has less plasticity. The result warrants further investigations for treatment strategies to enable ELM recovery. We have also shown the prognostic value of OCT parameters for visual outcome although the predictive power seemed inferior to that of initial visual acuity. Further development in the resolution of OCT images, identification of other factors, and/or a combination of the factors is needed to enhance this prognostic value.

Acknowledgement

Funding: The study was supported in part by a Grant-In-Aid for Scientific Research (No. 22791706) from the Japanese Society for the Promotion of Science, Tokyo. Financial disclosures: none. 
Recovery of OCT findings after the treatment of AMD

References

1. Coker JG, Duker JS (1996) Macular disease and optical coherence tomography. Curr Opin Ophthalmol 7: 33-38

2. Salinas-Alamán A, Garcia-Layana A, Maldonado MJ, Sainz-Gomez C, Alvarez-Vidal A (2005) Using optical coherence tomography to monitor photodynamic therapy in age related macular degeneration. Am J Ophthalmol 140: 23-28

3. Kashani AH, Keane PA, Dustin L, Walsh AC, Sadda SR (2009) Quantitative subanalysis of intraretinal cystoid spaces and outer nuclear layer using optical coherence tomography in neovascular age-related macular degeneration. Invest Ophthalmol Vis Sci 50: 3366-3373

4. Landa G, Bukelman A, Katz H, Pollack A (2009) Early OCT changes of neuroretinal foveal thickness after first versus repeated PDT in AMD. Int Ophthalmol 29: 1-5

5. Keane PA, Liakopoulos S, Jivrajka RV, Chang KT, Alasil T, Walsh AC, Sadda SR (2009) Evaluation of optical coherence tomography central retinal thickness parameters for use as anatomic outcomes in clinical trials for neovascular age-related macular degeneration. Invest Ophthalmol Vis Sci 50: 3378-3385

6. Drexler W, Sattmann H, Hermann B, Ko TH, Stur M, Unterhuber A, Scholda C, Findl O, Wirtitsch M, Fujimoto JG, Fercher AF (2003) Enhanced visualization of macular pathology with the use of ultrahigh-resolution optical coherence tomography. Arch Ophthalmol 121: 695-706

7. Ko TH, Fujimoto JG, Schuman JS, Paunescu LA, Kowalevicz AM, Hartl I, Drexler W, Wollstein G, Ishikawa H, Duker JS (2005) Comparison of ultrahigh- and standard-resolution optical coherence tomography for imaging macular pathology. Ophthalmology 112: 1922

8. Hayashi H, Yamashiro K, Tsujikawa A, Ota M, Otani A, Yoshimura N (2009) Association between foveal photoreceptor integrity and visual outcome in neovascular age-related macular degeneration. Am J Ophthalmol 148: 83-89

9. Sayanagi K, Sharma S, Kaiser PK (2009) Photoreceptor status after antivascular endothelial growth factor therapy in exudative age-related 
Recovery of OCT findings after the treatment of AMD

macular degeneration. $\mathrm{Br} \mathrm{J}$ Ophthalmol 93: 622-626

10. Landa G, Su E, Garcia PM, Seiple WH, Rosen RB (2011) Inner segment-outer segment junctional layer integrity and corresponding retinal sensitivity in dry and wet forms of age-related macular degeneration. Retina 31: 364-370

11. Oishi A, Hata M, Shimozono M, Mandai M, Nishida A, Kurimoto Y (2010) The significance of external limiting membrane status for visual acuity in age-related macular degeneration. Am J Ophthalmol 150: 27-32

12. Coscas G, Coscas F, Vismara S, Zourdani A, Li Calzi C (2009) OCT interpretation. In: Coscas G, Coscas F, S V, Zourdani A, Li Calzi C (eds) Optical coherence tomography in age-related macular degeneration. Springer-Verlag, Heidelberg, pp. 97-170.

13. Coscas G, Coscas F, Vismara S, Zourdani A, Li Calzi C (2009) Clinical features and natural history of AMD. In: Coscas G, Coscas F, S V, Zourdani A, Li Calzi C (eds) Optical coherence tomography in age-related macular degeneration. Springer-Verlag, Heidelberg, pp. 171-274.

14. Rosenfeld PJ, Brown DM, Heier JS, Boyer DS, Kaiser PK, Chung CY, Kim RY (2006) Ranibizumab for neovascular age-related macular degeneration. N Engl J Med 355: 1419-1431

15. Brown DM, Kaiser PK, Michels M, Soubrane G, Heier JS, Kim RY, Sy JP, Schneider S (2006) Ranibizumab versus verteporfin for neovascular age-related macular degeneration. N Engl J Med 355: 1432-1444

16. Brown DM, Michels M, Kaiser PK, Heier JS, Sy JP, Ianchulev T (2009) Ranibizumab versus verteporfin photodynamic therapy for neovascular age-related macular degeneration: Two-year results of the ANCHOR study. Ophthalmology 116: 57-65

17. Oishi A, Mandai M, Nishida A, Hata M, Matsuki T, Kurimoto Y (2011) Remission and dropout rate of anti-VEGF therapy for age-related macular degeneration. Eur J Ophthalmol 21: 777-782

18. Bressler NM (2009) Antiangiogenic approaches to age-related macular degeneration today. Ophthalmology 116: S15-23

19. Golbaz I, Ahlers C, Stock G, Schutze C, Schriefl S, Schlanitz F, Simader C, Prunte C, Schmidt-Erfurth UM (2011) Quantification of the therapeutic response of intraretinal, subretinal, and subpigment epithelial compartments in exudative AMD during anti-VEGF therapy. Invest 
Recovery of OCT findings after the treatment of AMD

Ophthalmol Vis Sci 52: 1599-1605

20. Brown DM, Regillo CD (2007) Anti-VEGF agents in the treatment of neovascular age-related macular degeneration: applying clinical trial results to the treatment of everyday patients. Am J Ophthalmol 144: 627-637

21. Sung $\mathrm{CH}$, Chuang JZ (2010) The cell biology of vision. J Cell Biol 190: 953-963

22. Shimozono M, Oishi A, Hata M, Kurimoto $Y$ (2011) Restoration of the photoreceptor outer segment and visual outcomes after macular hole closure: spectral-domain optical coherence tomography analysis. Graefes Arch Clin Exp Ophthalmol 249: 1469-1476

23. Chiang A, Chang LK, Yu F, Sarraf D (2008) Predictors of anti-VEGF-associated retinal pigment epithelial tear using FA and OCT analysis. Retina 28: 1265-1269

24. Moroz I, Moisseiev J, Alhalel A (2009) Optical coherence tomography predictors of retinal pigment epithelial tear following intravitreal bevacizumab injection. Ophthalmic Surg Lasers Imaging 40: 570-575

25. Singh RP, Fu EX, Smith SD, Williams DR, Kaiser PK (2009) Predictive factors of visual and anatomical outcome after intravitreal bevacizumab treatment of neovascular age-related macular degeneration: an optical coherence tomography study. Br J Ophthalmol 93: 1353-1358

26. Byun YJ, Lee SJ, Koh HJ (2010) Predictors of response after intravitreal bevacizumab injection for neovascular age-related macular degeneration. Jpn J Ophthalmol 54: 571-577

27. Unver YB, Yavuz GA, Bekiroglu N, Presti P, Li W, Sinclair SH (2009) Relationships between clinical measures of visual function and anatomic changes associated with bevacizumab treatment for choroidal neovascularization in age-related macular degeneration. Eye (Lond) 23: 453-460

28. Keane PA, Liakopoulos S, Chang KT, Wang M, Dustin L, Walsh AC, Sadda SR (2008) Relationship between optical coherence tomography retinal parameters and visual acuity in neovascular age-related macular degeneration. Ophthalmology 115: 2206-2214

29. Shimozono M, Oishi A, Hata M, Matsuki T, Ito S, Ishida K, Kurimoto $Y$ (2012) The Significance of Cone Outer Segment Tips as a Prognostic Factor in Epiretinal Membrane Surgery. Am J Ophthalmol DOI: 
Recovery of OCT findings after the treatment of AMD

S0002-9394(11)00719-7 [pii] 10.1016/j.ajo.2011.09.011 
Recovery of OCT findings after the treatment of AMD

Figure legends

Figure 1

Optical coherence tomography images of a patient with age-related macular degeneration demonstrating how the measurements were performed in the study. Length of External limiting membrane (ELM) and inner segment / outer segment junction (IS/OS) was measured along with each line as closely as possible using a caliper tool within a region $1 \mathrm{~mm}$ temporal and nasal from the fovea. In the presented case, both lines were disturbed where subretinal hemorrhage and fibrin existed (asterisk) and exact measurements were as follows ELM: from left to right 448, 224, 638, and $365 \mu \mathrm{m}$ IS/OS: 400, 225, 515, 72 , and $352 \mu \mathrm{m}$.

Figure 2

Pre- and post-operative changes in each parameter. Left: Foveal thickness decreased by approximately $30 \%$ and the change was statistically significant.

Right: While IS/OS recovered its continuity, the ELM length did not show significant improvement. * indicates $\mathrm{P}<0.001$

Figure 3

Pre- (upper) and post- (lower) operative OCT images of a representative case 
Recovery of OCT findings after the treatment of AMD

that showed improvement of IS/OS length. In the pre-operative image, IS/OS (arrow) was disturbed where intraretinal fluid accumulated. The post-operative image showed improvement of IS/OS, which was almost complete within $1 \mathrm{~mm}$ region from fovea.

Figure 4

Pre- (upper) and post- (lower) operative OCT images of a representative case that showed improvement of ELM length. Note the accumulation of subretinal fibrin and hard exudate in the pre-operative image (asterisk). ELM (triangle) was confirmed outside the deposits but is obscured in the central fovea where fibrin infiltrates into the outer retina. The post-operative image showed almost intact ELM in the depicted area. IS/OS was also improved in this case. We cannot tell whether the change represented the improvement of ELM or the removal of a signal-masking effect. 


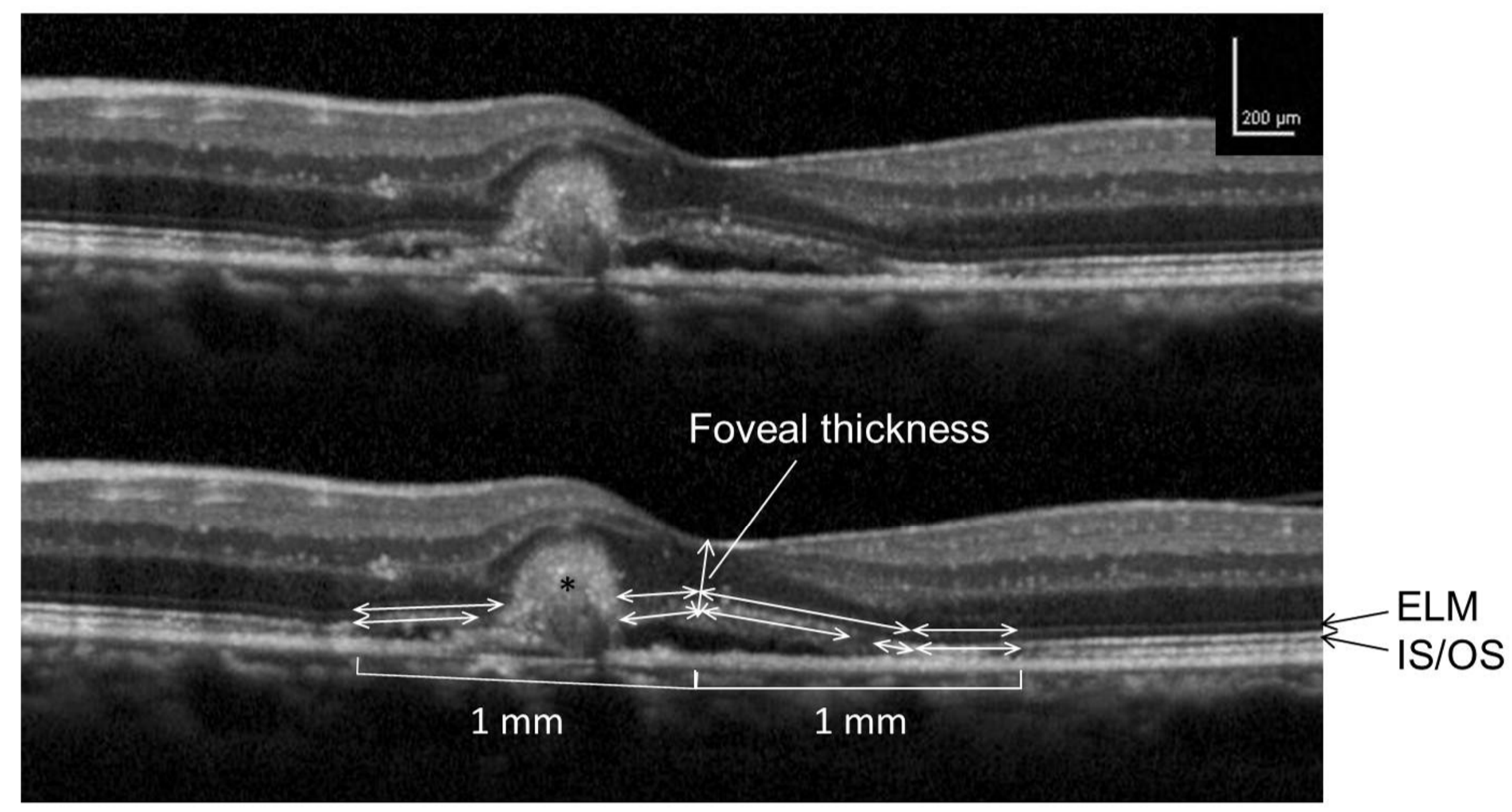

Figure 1 

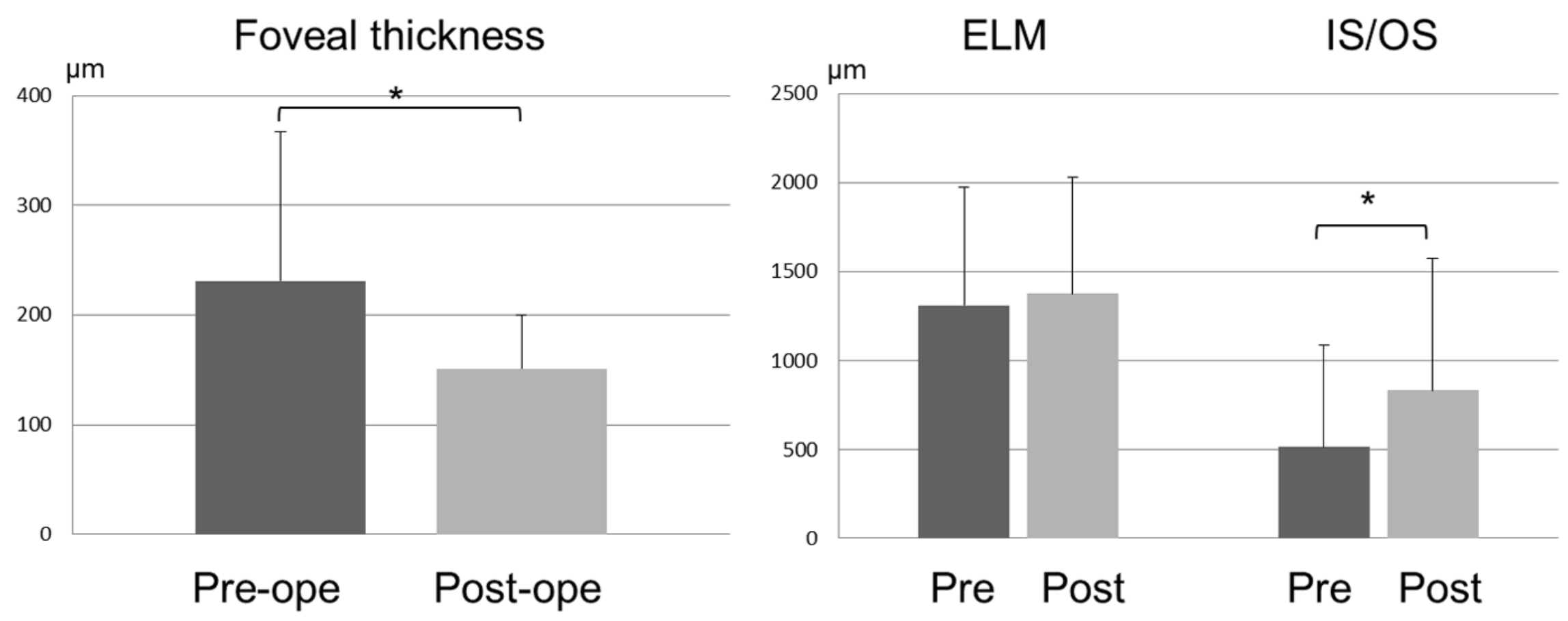

Figure 2 


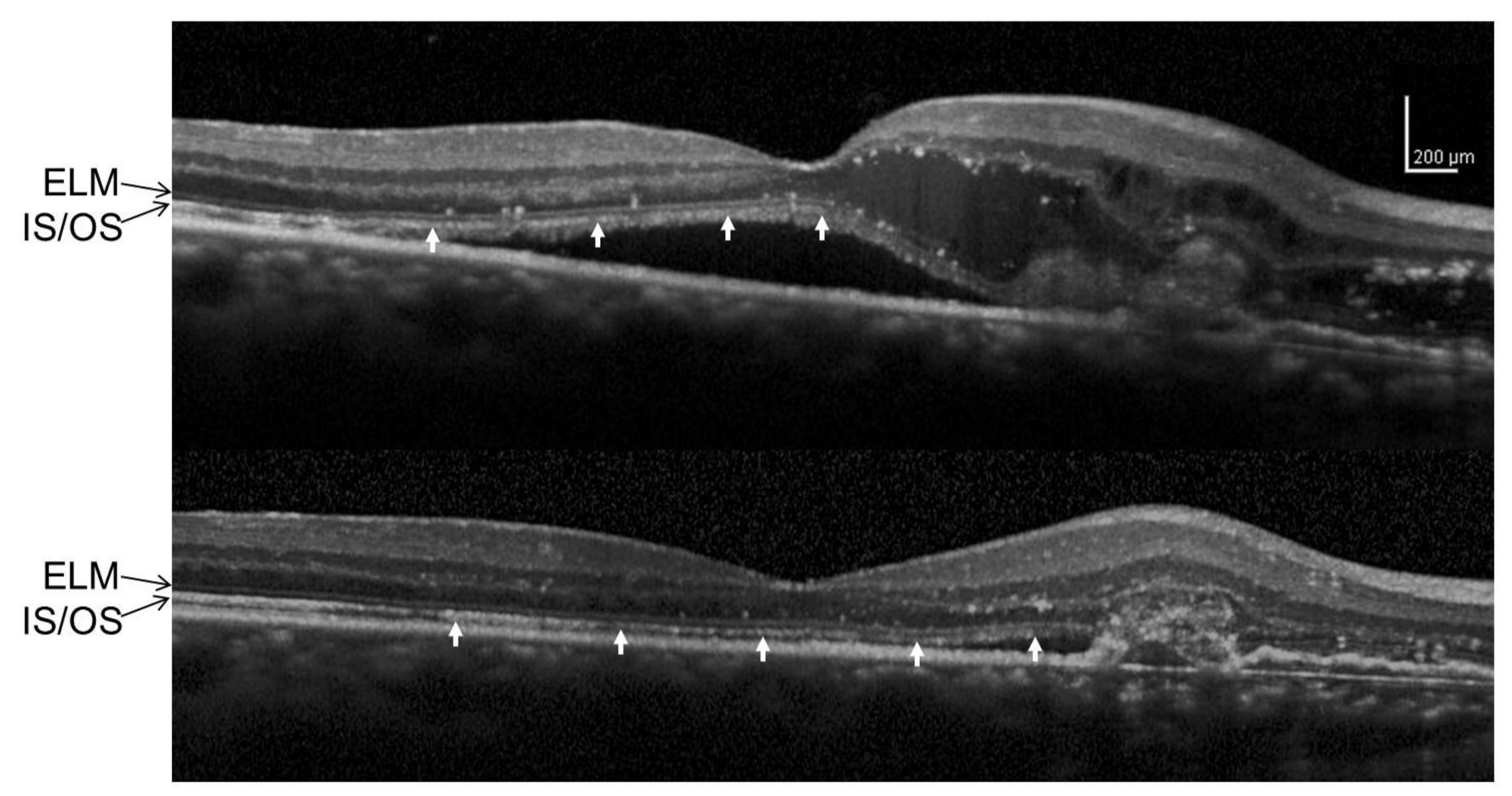

Figure 3 


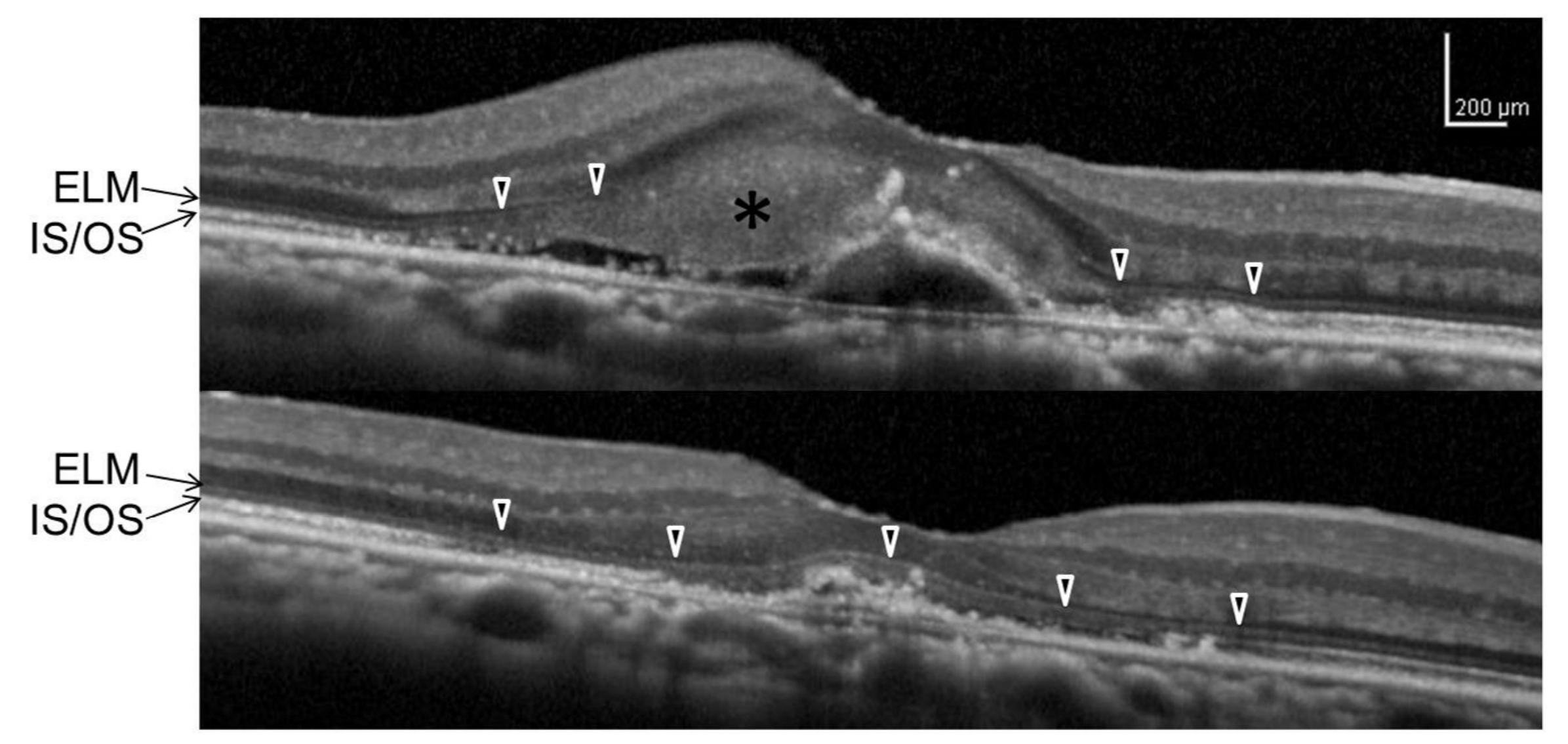

Figure 5 